\title{
AN EXPLICIT FORMULA FOR THE PICARD GROUP OF THE CYCLIC GROUP OF ORDER $p^{2}$
}

\author{
ALEXANDER STOLIN \\ (Communicated by Eric Friedlander)
}

\begin{abstract}
We give a formula for the Picard group of the integer group ring of the cyclic group of order $p^{2}$ for any odd prime $p$. As a corollary one gets a formula for properly irregular prime $p$ in terms of Bernoulli numbers.
\end{abstract}

\section{INTRODUCTION}

Let $C_{p^{n}}$ be a cyclic $p$-group of order $p^{n}$, where $p$ is an odd prime number, and let $\zeta_{n}$ be a primitive $p^{n}$ th root of unity. The Picard group of integer group rings $\mathbb{Z}\left[C_{p^{n}}\right]$ were intensively studied in several papers (see [V, K-M, G, S1, S2]). The results of these investigations are summarized in [C-R], but one point that is very essential for our investigation is not mentioned there. In [U], Ullom obtained an algorithm giving the possibility of computing the Picard group for groups $C_{p^{2}}$, when $p$ is a properly irregular prime. It is not difficult to notice that in the cases treated in [U] the class group $\mathrm{Cl}\left(\mathbb{Z}\left[\zeta_{2}\right]\right)$ is a direct summond in $\operatorname{Pic}\left(\mathbb{Z}\left[C_{p^{2}}\right]\right)$.

The purpose of the present paper is to prove this fact for arbitrary primes $p$. Combining this with a result concerning a property of integers in cyclotomic fields $\mathbb{Q}\left(\zeta_{n}\right)$, we obtain an explicit formula for $\operatorname{Pic}\left(\mathbb{Z}\left[C_{p^{2}}\right]\right)$ without restriction on $p$. As a special case, we reprove Ullom's result expressing $\operatorname{Pic}\left(\mathbb{Z}\left[C_{p^{2}}\right]\right)$ for properly irregular primes in terms of Bernoulli numbers using a different method.

Some of our results can be generalized. Denoting by $\zeta_{n, k}$ the class $x$ in $\mathbb{Z}[x] /\left(\Phi_{n, k}(x)\right)=\mathbb{Z}\left[\zeta_{n, k}\right]$ where $\Phi_{n, k}(x)=\left(x^{p^{n}}-1\right) /\left(x^{p^{k}}-1\right)$ for $0 \leq k \leq n$, we are able to prove that $\mathrm{Cl} \mathbb{Z}\left[\zeta_{n}\right]$ is a direct summond in $\operatorname{Pic}\left(\mathbb{Z}\left[\zeta_{n, k}\right]\right)$ for any $k$ and any prime number $p$. (See [S3].)

The paper consists of two sections. In the first, we prove a number of auxiliary results and, in particular, a lemma on integers in cyclotomic fields, which plays a key role in the paper. In the second section, we apply the lemma to a suitable Mayer-Vietoris sequence in order to compute the group Pic $\mathbb{Z}\left[C_{p^{2}}\right]$ for arbitrary odd prime number $p$.

Received by the editors September 25, 1992.

1991 Mathematics Subject Classification. Primary 11R18, 11R33, 19A31.

This paper was supported by the Feinberg Graduate School. 


\section{Auxiliary Results}

In this section, we prove a number of technical results, which we use in the computations of Picard groups. The key point is Lemma 2, which will be used twice in the next section. But we start with a well-known result, whose proof is included for the convenience of the reader. We keep the notations previously introduced.

Proposition 1. $\operatorname{Pic} \mathbb{Z}\left[C_{p^{n}}\right] \equiv \operatorname{Pic} \mathbb{Z}\left[\zeta_{n, 0}\right]$.

Proof. We have $\mathbb{Z}\left[C_{p^{n}}\right]=\mathbb{Z}[x] /\left(x^{p^{n}}-1\right)$. Let us consider the following Cartesian square with obvious homomorphisms:

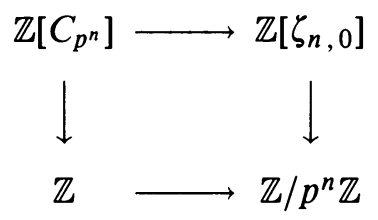

According to $[\mathrm{M}]$, we get the following Mayer-Vietoris exact sequence:

$$
\begin{aligned}
U\left(\mathbb{Z}\left[C_{p^{n}}\right]\right. & \rightarrow U(\mathbb{Z}) \oplus U\left(\mathbb{Z}\left[\zeta_{n, 0}\right] \rightarrow U\left(\mathbb{Z} / p^{n} \mathbb{Z}\right) \rightarrow \operatorname{Pic} \mathbb{Z}\left[C_{p^{n}}\right]\right. \\
& \rightarrow \operatorname{Pic} \mathbb{Z} \oplus \operatorname{Pic} \mathbb{Z}\left[\zeta_{n, 0}\right] \rightarrow \operatorname{Pic} \mathbb{Z} / p^{n} \mathbb{Z} .
\end{aligned}
$$

Since $\operatorname{Pic} \mathbb{Z}=\operatorname{Pic} \mathbb{Z} / p^{n} \mathbb{Z}=0$, it suffices to show that $U\left(\mathbb{Z}\left[\zeta_{n}, 0\right]\right) \rightarrow U\left(\mathbb{Z} / p^{n} \mathbb{Z}\right)$ is an epimorphism. Let us consider the element $\left(\zeta_{n, 0}^{k}-1\right) /\left(\zeta_{n, 0}-1\right) \in \mathbb{Z}\left[\zeta_{n, 0}\right]$ such that $(k, p)=1$.

Choose $r$ such that $t=1+p^{n} r$ is divisible by $k$. Then

$$
\frac{\zeta_{n, 0}^{k}-1}{\zeta_{n, 0}-1} \cdot \frac{\zeta_{n, 0}^{1+p^{n} r}-1}{\zeta_{n, 0}^{k}-1}=1
$$

and hence,

$$
\varepsilon_{k}=\frac{\zeta_{n, 0}^{k}-1}{\zeta_{n, 0}-1} \in U\left(\mathbb{Z}\left[\zeta_{n, 0}\right]\right) .
$$

It is clear that $j\left(\varepsilon_{k}\right)=k$, so the proof is complete.

Now let us consider the Cartesian square

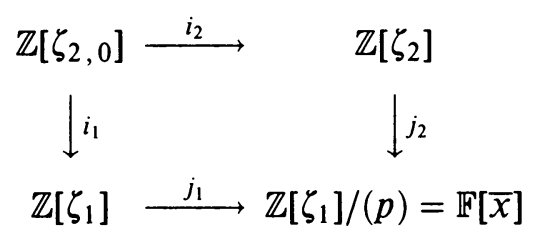

where $\bar{x}$ is the residue of $x$ in $\mathbb{F}_{p}[x] /(x-1)^{p-1}, i_{k}\left(\zeta_{2,0}\right)=\zeta_{k}$, and $j_{k}\left(\zeta_{k}\right)=\bar{x}$ for $k=1,2$ and the corresponding exact Mayer-Vietoris sequence

$$
\begin{aligned}
U\left(\mathbb{Z}\left[\zeta_{2}, 0\right]\right) & \rightarrow U\left(\mathbb{Z}\left[\zeta_{1}\right]\right) \oplus U\left(\mathbb{Z}\left[\zeta_{2}\right]\right) \rightarrow U\left(\mathbb{Z}\left[\zeta_{1}\right] /(p)\right) \\
& \rightarrow \operatorname{Pic} \mathbb{Z}\left[\zeta_{2,0}\right] \rightarrow \mathrm{Cl} \mathbb{Z}\left[\zeta_{1}\right] \oplus \mathrm{Cl} \mathbb{Z}\left[\zeta_{2}\right] \rightarrow 0
\end{aligned}
$$

Denote by $N$ the norm map $N: \mathbb{Q}\left(\zeta_{2}\right) \rightarrow \mathbb{Q}\left(\zeta_{1}\right)$.

The following result is proved in [C-R]. We give a different proof, which will be more convenient for the applications we have in mind. 
Lemma 1. Keeping the notation in $(*)$, the following diagram is commutative:

$$
\mathbb{Z}\left[\zeta_{2}\right]
$$

$$
\begin{array}{cc}
N \swarrow & \searrow j_{2} \\
\mathbb{Z}\left[\zeta_{1}\right] \stackrel{j_{1}}{\longrightarrow} \mathbb{Z}\left[\zeta_{1}\right] /(p)
\end{array}
$$

Proof. We have $N\left(\zeta_{2}\right)=\zeta_{1}$, and therefore, it suffices to prove that $N(a+b) \equiv$ $N(a)+N(b) \bmod p$. Since $\mathbb{Z}\left[\zeta_{2}\right]$ is free as a $\mathbb{Z}\left[\zeta_{1}\right] /(p)$-module, $\mathbb{Z}\left[\zeta_{2}\right] /(p)$ is free as a $\mathbb{Z}\left[\zeta_{1}\right] /(p)$-module. Hence, $N$ induces a well-defined mapping $\tilde{N}: \mathbb{Z}\left[\zeta_{2}\right] /(p) \rightarrow \mathbb{Z}\left[\zeta_{1}\right] /(p)$, and it is sufficient to prove that $\tilde{N}(a)=a^{p}$. Since $\mathbb{Z}\left[\zeta_{2}\right] /(p) \cong \mathbb{F}_{p}[x] /\left((x-1)^{p^{2}-p}\right) \cong \mathbb{F}_{p}[z] /\left(z^{p^{2}-p}\right)$, where $z=x-1$, and $\mathbb{Z}\left[\zeta_{1}\right] /(p) \cong \mathbb{F}_{p}[y] /\left(y^{p-1}\right)$, we can reduce our problem to the ring extension $\mathbb{F}_{p}[y] \subset \mathbb{F}[z]$, where $z^{p}=y$. It is well known that in this case the norm map $N: \mathbb{F}_{p}[z] \rightarrow \mathbb{F}_{p}[y]$ is given by the formula $N r(\alpha)=\alpha^{p}$ for any $\alpha \in \mathbb{F}_{p}[z]$. This observation completes the proof.

The following result will play a crucial role in the sequel:

Lemma 2. Let $q \in \mathbb{Z}\left[\zeta_{n}\right]$, where $\zeta_{n}^{p^{n}}=1$. Let also $q \equiv 1 \bmod \left(1-\zeta_{n}\right)^{p^{n}-1}$ and $(q)=I^{p}$ for some ideal $I$ in $\mathbb{Z}\left[\zeta_{n}\right]$. Then $q \equiv 1 \bmod \left(1-\zeta_{n}\right)^{p^{n}}$.

Proof. First of all, let us shortly recall some properties of the norm residue symbol (see [C-F]).

Let $K=\mathbb{Q}\left(\zeta_{n}\right)$. For any valuation $\omega$ of $K$ and any $a \in K^{*}$ we have the local Artin map $\psi_{w}: K_{w}^{*} \rightarrow \operatorname{Gal}\left(K_{\omega}(p \sqrt{a}) / K_{\omega}\right)$. Using Kummer's pairing, the norm residue symbol $(a, b)_{\omega}$ is defined as follows: $(a, b)_{\omega}=(p \sqrt{a})^{\psi_{\omega}(b)} \cdot(p \sqrt{a})^{-1}$ with values in the group of $p$ th roots of unity.

We need the following properties of the symbol:

(a) $\Pi_{\omega}(a, b)_{\omega}=1$, where the product is over all valuations of $K$.

(b) If $a+b \in\left(K_{\omega}^{*}\right)^{p}$, then $(a, b)_{\omega}=1$.

(c) $(a, b)_{\omega}=1$ if and only if $b$ is a norm from the extension $K_{\omega}(p \sqrt{a}) / K_{\omega}$.

(d) Let $v=\left(1-\zeta_{n}\right)$ be a valuation of $K$. Let $\lambda=1-\zeta_{n}$ and $\eta_{i}=1-\lambda^{i}$.

Then $\left(\eta_{i}, \eta_{j}\right)_{v}=1$ if $i+j>p^{n}$ and $\left(\eta_{i}, \eta_{j}\right)_{v} \neq 1$ if $i+j=p^{n}$ and $1 \leq i<p$.

Now we are ready to prove the lemma.

Step I. Let $q$ satisfy the conditions of the lemma and $\varepsilon \in \mathbb{Z}\left[\zeta_{n}\right]$. Then $(\varepsilon, q)_{v}=1$, where $v=\left(1-\zeta_{n}\right)$. Indeed, for $\omega \neq v$ the extension $K_{\omega}(p \sqrt{q}) / K_{\omega}$ is nonramified, and therefore $\varepsilon$ is a norm in this extension. Thus, according to the property $(\mathrm{c}),(\varepsilon, q)_{\omega}=1$ for $\omega \neq v$. Then property (a) implies that $(\varepsilon, q)_{v}=1$.

Step II. Let $\varepsilon=\zeta_{n}=1-\lambda=\eta_{1}$ (see $\left.(\mathrm{d})\right)$. Then $\left(\eta_{1}, q\right)_{v}=1$ by Step I. On the other hand $\left(\eta_{1}, q\right)_{v} \neq 1$ if $q \equiv 1 \bmod \left(1-\zeta_{n}\right)^{p^{n}-1}$ and $q \neq 1 \bmod \left(1-\zeta_{n}\right)^{p_{n}}$ (implied by properties (b) and (d)). Thus, $q \equiv 1 \bmod \left(1-\zeta_{n}\right)^{p^{n}}$ as required.

We now need some notation on the structure of nonramified abelian extensions of period $p$ over $K_{1}=\mathbb{Q}\left(\zeta_{1}\right)$.

(1) Let $S=\mathrm{Cl} \mathbb{Z}\left[\zeta_{1}\right]$. Then the Galois group of the maximal nonramified extension of the period $p$ over $K_{1}$ is isomorphic to $S / S^{p}$.

(2) Kummer's theory implies that $\operatorname{Char}\left(S / S^{p}\right)$ is generated by $q \in K_{1}^{*}$ satisfying the following conditions: $(q)=I^{p}, q \equiv 1 \bmod \left(1-\zeta_{1}\right)^{p}$. 
(3) Let $\varepsilon \in U\left(\mathbb{Z}\left[z_{1}\right]\right)$ satisfy the condition $\varepsilon \equiv 1 \bmod \left(1-\zeta_{1}\right)^{p}$. Obviously in this case $\varepsilon \equiv 1 \bmod \left(1-\zeta_{1}\right)^{p+1}$ since $\varepsilon$ is a real unit by Kummer's Lemma and $p$ is odd. Then the extension $K_{1}(p \sqrt{\varepsilon}) / K_{1}$ is nonramified and $\varepsilon$ induces the character of the group $S / S^{p}$. Let us denote the subgroup of the group $\operatorname{Char}\left(S / S^{p}\right)$, generated by all such $\varepsilon \in U\left(\mathbb{Z}\left[\zeta_{1}\right]\right)$, by $\left(\operatorname{Char} S / S^{p}\right)_{\varepsilon}$.

Corollary 1. For $r=\log _{p}\left(\#\left(\mathrm{Char} S / S^{p}\right)_{\varepsilon}\right)$, we have the following short exact sequence:

0 $(\mathbb{Z} / p \mathbb{Z})^{(p-3) / 2+r}$ $\operatorname{Pic} \mathbb{Z}\left[\zeta_{2}, 0\right]$ $\operatorname{Pic} \mathbb{Z}\left[\zeta_{1}\right] \oplus \operatorname{Pic} \mathbb{Z}\left[\zeta_{2}\right]$ 0

Proof. Let us consider the following Cartesian square:
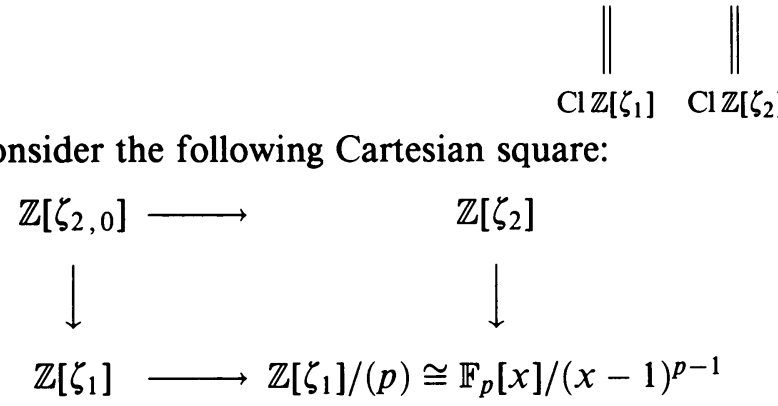

Then we have the exact Mayer-Vietoris sequence

$$
\begin{aligned}
U\left(\mathbb{Z}\left[\zeta_{2}\right]\right) \oplus U\left(\mathbb{Z}\left[\zeta_{1}\right]\right) & \rightarrow U\left(\mathbb{Z}\left[\zeta_{1}\right] /(p)\right) \\
& \rightarrow \operatorname{Pic} \mathbb{Z}\left[\zeta_{2}, 0\right] \rightarrow \operatorname{Pic} \mathbb{Z}\left[\zeta_{1}\right] \oplus \operatorname{Pic} \mathbb{Z}\left[\zeta_{2}\right] \rightarrow 0
\end{aligned}
$$

Since the diagram

$$
\mathbb{Z}\left[\zeta_{2}\right]
$$

is commutative,

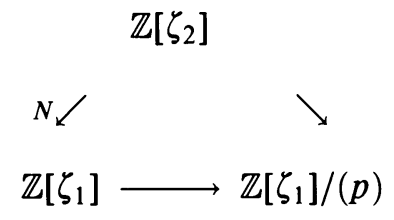

$$
\operatorname{Im} U\left(\mathbb{Z}\left[\zeta_{2}\right]\right) \subset \operatorname{Im} U\left(\mathbb{Z}\left[\zeta_{1}\right]\right) .
$$

The structure of $U\left(\mathbb{Z}\left[\zeta_{1}\right] /(p)\right)$ is well known:

$$
U\left(\mathbb{Z}\left[\zeta_{1}\right] /(p)\right) \cong \mathbb{F}_{p}^{*} \oplus(\mathbb{Z} / p \mathbb{Z})^{p-2} .
$$

Therefore, to convert the long sequence to the short one, it suffices to find the image of $U\left(\mathbb{Z}\left[\zeta_{1}\right]\right)$ in $U\left(\mathbb{Z}\left[\zeta_{1}\right] /(p)\right)$, i.e.,

$$
U\left(\mathbb{Z}\left[\zeta_{1}\right]\right) /\{\varepsilon \equiv 1 \bmod p\} .
$$

Obviously, $\{\varepsilon \equiv 1 \bmod p\} \supset\left\{\gamma \equiv 1 \bmod \left(1-\zeta_{1}\right)\right\}^{p}$. By the theorem we have proved

$$
U\left(\mathbb{Z}\left[\zeta_{1}\right]\right) /\{\varepsilon \equiv 1 \bmod p\}=\frac{\left(U\left(\mathbb{Z}\left[\zeta_{1}\right]\right) /\left\{\gamma \equiv 1 \bmod \left(1-\zeta_{1}\right)\right\}^{p}\right)}{\left(\left\{\varepsilon \equiv 1 \bmod \left(1-\zeta_{1}\right)^{p+1}\right\} /\{\gamma \equiv 1\}^{p}\right)} .
$$

We have

(1) by Kummer's Lemma $U\left(\mathbb{Z}\left[\zeta_{1}\right]\right) \cong\left\langle\zeta_{1}\right\rangle \oplus\langle$ real units $\rangle \cong \mathbb{Z} / p \mathbb{Z} \oplus\left(\mathbb{Z}^{(p-3) / 2}\right)$.

(2) $\left\{\varepsilon \equiv 1 \bmod \left(1-\zeta_{1}\right)^{p+1}\right\} /\left\{\gamma \equiv 1 \bmod \left(1-\zeta_{1}\right)\right\}^{p} \cong\left(\operatorname{Char} S / S^{p}\right)_{\varepsilon} \cong$ $(\mathbb{Z} / p \mathbb{Z})^{r}$. 
Since (1) implies that $U\left(\mathbb{Z}\left[\zeta_{1}\right]\right) /\left\{\gamma \equiv 1 \bmod \left(1-\zeta_{1}\right)\right\}^{p} \cong \mathbb{F}_{p}^{*} \oplus(\mathbb{Z} / p \mathbb{Z}) \oplus$ $(\mathbb{Z} / p \mathbb{Z})^{(p-3) / 2}$, we have $U\left(\mathbb{Z}\left[\zeta_{1}\right]\right) /\{\varepsilon \equiv 1 \bmod p\} \cong \mathbb{F}_{p}^{*} \oplus(\mathbb{Z} / p \mathbb{Z})^{(p-1) / 2-r}$ and, hence,

$$
U\left(\mathbb{Z}\left[\zeta_{1}\right] /(p)\right) / \operatorname{Im}\left(\mathbb{Z}\left[\zeta_{1}\right]\right) \cong(\mathbb{Z} / p \mathbb{Z})^{p-2-(p-1) / 2+r}=(\mathbb{Z} / p \mathbb{Z})^{(p-3) / 2+r} .
$$

Corollary 2. (1) $U\left(\mathbb{Z}\left[\zeta_{2,0}\right] \cong U\left(\mathbb{Z}\left[\zeta_{2}\right]\right) \oplus U\left(\mathbb{Z}\left[\zeta_{1}\right]\right)_{p-1}\right.$ where $U\left(\mathbb{Z}\left[\zeta_{1}\right]\right)_{p-1}=\{\varepsilon \in$ $\left.U\left(\mathbb{Z}\left[\zeta_{1}\right]\right): \varepsilon \equiv 1 \bmod p\right\}$.

(2) $U\left(\mathbb{Z}\left[\zeta_{1}\right]\right)_{p-1} / U\left(\mathbb{Z}\left[\zeta_{1}\right]\right)^{p} \cong(\mathbb{Z} / p \mathbb{Z})^{r}$, where $r=\log _{p} \#\left\{\left(\operatorname{Char} S / S^{p}\right)_{\varepsilon}\right\}$.

Proof. (1) Obviously, $U\left(\mathbb{Z}\left[\zeta_{2, p}\right]\right)=\left\{\left(\varepsilon_{1}, \varepsilon_{2}\right): \varepsilon_{i} \in U\left(\mathbb{Z}\left[\zeta_{i}\right]\right), j_{1}\left(\varepsilon_{1}\right)=j_{2}\left(\varepsilon_{2}\right)\right\}$. Then $\left(\varepsilon_{1}, \varepsilon_{2}\right)=\left(N\left(\varepsilon_{2}\right), \varepsilon_{2}\right) \cdot\left(\varepsilon_{1} \cdot N\left(\varepsilon_{2}^{-1}\right), 1\right)$. This gives what was required.

(2) It has been proved in the proof of Corollary 1.

Remark. If $p$ is properly irregular, then $r=\#$ \{Bernulli numbers divisible by $p$ in the series $\left.B_{2}, B_{4}, \ldots, B_{p-3}\right\}$. (See [C-R, U, K-M, G, B-Sh, S1, S2].)

2

Lemma 1. Let $I$ be an ideal in a Dedekind ring $R$, and let $j: R \rightarrow R / I=\bar{R}$ be the natural projection, let $I_{1}$ and $I_{2}$ be ideals of $R$ co-prime to $I$, and let $h \in\left(I_{2}: I_{1}\right)=\operatorname{Hom}_{R}\left(I_{1}, I_{2}\right)$. Then there exists $x_{1} \in I_{1} \otimes_{R} \bar{R} \cong I_{1} / I \cdot I_{1}$ and $x_{2} \in I_{2} \otimes_{2} \bar{R} \cong I_{2} / I \cdot I_{2}$ such that

(1) $x_{1}$ (respectively $x_{2}$ ) is the basis element of the free module $I_{1} \otimes_{R} \bar{R}$ (respectively $I_{2} \otimes_{R} \bar{R}$ ) of rank 1 over $\bar{R}$.

(2) $h \otimes \mathrm{id}_{\bar{R}}: I_{1} \otimes_{R} \bar{R} \rightarrow I_{2} \otimes_{R} \bar{R}$ is determined by the element $j(h) \in \bar{R}$ with respect to $x_{1}$ and $x_{2}$. Here $\left(h \otimes \mathrm{id}_{\bar{R}}\right)\left(i_{1} \otimes \bar{r}\right)=h\left(i_{1}\right) \otimes \bar{r}$ for $i_{1} \in I_{1}$, $\bar{r} \in \bar{R}$.

Proof. Since $I+I_{1}=I+I_{2}=R$, we can choose $y_{1}, y_{2} \in I$ and $a_{1} \in I_{1}$ (respectively $a_{2} \in I_{2}$ ) such that $a_{1}+y_{1}=a_{2}+y_{2}=1$. Then we can put

$$
x_{1}=a_{1} \otimes_{R} 1_{\bar{R}} \in I_{1} \otimes_{R} \bar{R}, \quad x_{2}=a_{2} \otimes_{R} 1_{\bar{R}} .
$$

Let a Cartesian square of commutative rings

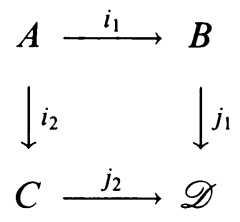

satisfy the following conditions:

(I) $B$ is a Dedekind ring.

(II) $j_{1}$ is an epimorphism.

(III) $\mathscr{D}$ is a local ring.

(IV) $C$ is such that the following property holds: for any projective module $P$ over $C$ and any $\varepsilon \in U(C)$ there exists an automorphism of $P$ with determinant $\varepsilon$.

Remark. Let $P$ be a projective module over $C$ and $\alpha$ be its automorphism. Let $Q$ be such that $P \oplus Q$ is free. Then $\alpha \oplus \mathrm{id}_{Q}$ is an automorphism of the free module $P \oplus Q$ and $\operatorname{det} \alpha \stackrel{\text { def }}{\rightarrow} \operatorname{det}\left(\alpha \oplus \operatorname{id}_{Q}\right) \in U(C)$. It is easy to see that $\operatorname{det} \alpha$ does not depend on $Q$. 
Lemma 2. Let a Cartesian square satisfy conditions (I)-(IV). Let $M\left(P_{1}, P_{2}, h\right)$ and $M\left(P_{1}, P_{2}, q\right)$ also be projective modules over $A$ (see the definition of $M\left(P_{1}, P_{2}, h\right)$ in [M] $)$. Then $M\left(P_{1}, P_{2}, h\right) \cong M\left(P_{1}, P_{2}, q\right)$ if and only if $\operatorname{det}\left(h^{-1} g\right)=\varepsilon_{1} \cdot \varepsilon_{2}$, where $\varepsilon_{1}, \varepsilon_{2} \in U(\mathscr{D})$ and $\varepsilon_{1}$ (respectively $\varepsilon_{2}$ ) can be lifted to $U(B)$ (respectively $U(C)$ ).

Proof. First of all let us notice that the projective module $M\left(P_{1}, P_{2}, h\right)$ is isomorphic to the projective module $M\left(P_{1}, P_{2}, g\right)$ if and only if there exists $t_{1} \in \operatorname{Aut}_{B}\left(P_{1}\right)$ and $t_{2} \in \operatorname{Aut}_{C}\left(P_{2}\right)$ such that the diagram

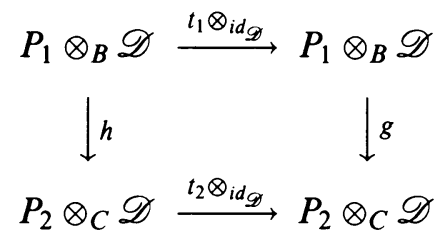

is commutative, where $\left(t_{i} \otimes_{\mathrm{id}_{\mathscr{D}}}\right)\left(p_{i} \otimes d\right)=t_{i}\left(p_{i}\right) \otimes d$ for $p_{i} \subset P_{i}, d \in \mathscr{D}$.

Now if we put $\varepsilon_{i}=\operatorname{det}\left(t_{i} \otimes \mathrm{id}_{\mathscr{D}}\right)$, then we obtain $\operatorname{det}\left(h^{-1} g\right)=\varepsilon_{1}^{-1} \varepsilon_{2}$.

Conversely: to given $h, g, \varepsilon_{1}, \varepsilon_{2}$ we must construct $t_{1}$ and $t_{2}$. Since $B$ also satisfies condition (IV), it suffices to prove the statement for $\varepsilon_{1}=\varepsilon_{2}=$ 1. Then we can put $t_{2}=\mathrm{id}_{P_{2}}$. Thus it remains to be proved that for any automorphism $f$ of the free module $P_{1} \otimes_{B} \mathscr{D}$ over $\mathscr{D}$ with $\operatorname{det} f=1$ there exists an automorphism $t_{1}$ of the module $P_{1}$ such that $f=t_{1} \otimes \mathrm{id}_{\mathscr{D}}$. Using the fact that every automorphism of a free module over a local ring can be decomposed in a product of elementary ones, we can reduce our problem to the following assertion:

Assertion. Let $S_{1}$ and $S_{2}$ be projective modules of rank 1 over $B$ and $k \in$ $\operatorname{Hom}_{\mathscr{D}}\left(S_{1} \otimes_{B} \mathscr{D}, S_{2} \otimes_{B} \mathscr{D}\right)$, where $B$ and $\mathscr{D}$ are as above. Then there exists $t \in \operatorname{Hom}_{B}\left(S_{1}, S_{2}\right)$ such that $k=t \otimes \operatorname{id}_{\mathscr{D}}$.

Clearly, Lemma 1 and condition (II) imply this assertion and that completes the proof of Lemma 2.

The following three corollaries give us a basis for the computation of $\operatorname{Pic} \mathbb{Z}\left[\zeta_{2,0}\right]$. First of all let us notice that the following square of the epimorphisms of the rings is a Cartesian and that it satisfies conditions (I)-(IV):

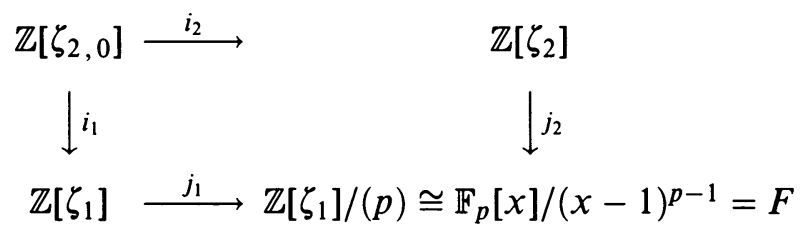

where $i_{k}\left(\zeta_{2}, 0\right)=\zeta_{k}, j_{k}\left(\zeta_{k}\right)=x, k=1,2$.

Corollary 1. Let $\alpha_{i}$ be an ideal in $\mathbb{Z}\left[\zeta_{i}\right](i=1,2)$ co-prime with $p$ and let $M\left(\alpha_{1}, \alpha_{2}, h\right)$ be a projective module over $\mathbb{Z}\left[\zeta_{2}, 0\right]$. Here

$$
h \in \operatorname{Hom}_{F}\left(\alpha_{1} \otimes_{\mathbb{Z}\left[\zeta_{1}\right]} F, \alpha_{2} \otimes_{\mathbb{Z}\left[\zeta_{2}\right]} F\right) \cong F
$$

is given with respect to $x_{1}, x_{2}$ such that $x_{i} \in \alpha_{i} \otimes_{\mathbb{Z}\left[\zeta_{1}\right]} F(i=1,2)$ and $x_{1}$ satisfies Lemma 1 with $R=\mathbb{Z}\left[\zeta_{1}\right], I=(p)$ and $x_{2}$ satisfies Lemma 1 with $R=\mathbb{Z}\left[\zeta_{2}\right], I=\left(1-\zeta_{2}\right)^{p-1}$. Then 
(1) $M\left(\alpha_{1}, \alpha_{2}, h\right) \cong M\left(q_{1} \alpha_{1}, q_{2} \alpha_{2}, j_{1}\left(q_{q}^{-1}\right) \cdot j_{2}\left(q_{2}\right) \cdot h\right)$, where $q_{i} \in \mathbb{Z}\left[\zeta_{i}\right]$ $(i=1,2),\left(q_{i}, p\right)=1$, and $j_{1}\left(q_{1}^{-1}\right) \cdot j_{2}\left(q_{2}\right)=\cdot h$ determines the element if $\operatorname{Hom}_{F}\left(q_{1} \alpha_{1} \otimes_{\mathbb{Z}\left[\zeta_{1}\right]} F, q_{2} \alpha_{2} \otimes_{\mathbb{Z}\left[\zeta_{2}\right]} F \cong F\right.$ with respect to $y_{1}, y_{2}$ such that $y_{i} \in q_{i} \alpha_{i} \otimes_{\mathbb{Z}\left[\zeta_{i}\right]} F(i=1,2)$ and $y_{1}$ satisfies Lemma 1 with $R=\mathbb{Z}\left[\zeta_{1}\right], I=(p)$ and $y_{2}$ satisfies Lemma 1 with $R=\mathbb{Z}\left[\zeta_{2}\right], I=\left(1-\zeta_{2}\right)^{p-1}$.

(2) $M\left(\alpha_{1}, \alpha_{2}, h\right)$ is free if and only if $\alpha_{i}=\left(r_{i}\right)$, where $r_{i} \in \mathbb{Z}\left[\zeta_{i}\right](i=1,2)$ and $j_{2}\left(r_{2}^{-1}\right) \cdot j_{1}\left(r_{1}\right) \cdot h=j_{1}(\omega)$ for some $\omega \in U\left(\mathbb{Z}\left[\zeta_{1}\right]\right)$.

Proof. To prove the first statement we proceed just as in the proof of Lemma 2. The second statement follows from the first one and Lemma 1 from $\S 1$.

Remark. In the remaining part of the paper, writing $M\left(\alpha_{1}, \alpha_{2}, h\right)$ we will suppose that $\alpha_{i} \subset \mathbb{Z}\left[\zeta_{i}\right](i=1,2)$ are co-prime with $p$ and $h$ given in the same way as in Corollary 1.

Corollary 2. (1) $M\left(\alpha_{1}, \alpha_{2}, h\right) \oplus M\left(p_{1}, p_{2}, g\right) \cong M\left(\alpha_{1} p_{1}, \alpha_{2} p_{2}, h g\right) \oplus \mathbb{Z}\left[\zeta_{2,0}\right]$.

(2) $M\left(\alpha_{1}, \alpha_{2}, h\right) \otimes M\left(p_{1}, p_{2}, g\right) \cong M\left(\alpha_{1} p_{1}, \alpha_{2} p_{2}, h g\right)$.

Proof. The first equality holds due to $\alpha_{i} \oplus p_{i} \cong \alpha_{i} p_{i} \oplus \mathbb{Z}\left[\zeta_{i}\right](i=1,2)$. The second holds due to the first and the existence of the functor det: $K_{0} \rightarrow$ Pic (see [B]).

Corollary 3. Let $\alpha$ be an ideal in $\mathbb{Z}\left[\zeta_{2}\right]$ co-prime with $p$. Let us also denote by $N$ the map $N: I\left(\mathbb{Z}\left[\zeta_{2}\right]\right) \rightarrow I\left(\mathbb{Z}\left[\zeta_{1}\right]\right)$, where $I\left(\mathbb{Z}\left[\zeta_{i}\right]\right)$ is the group of ideals of the ring $\mathbb{Z}\left[\zeta_{i}\right]$ (see [B-Sh]). Then the map $\varphi: \alpha \rightarrow M(N(\alpha), \alpha, 1)$ determines a homomorphism $\varphi: \mathrm{Cl} \mathbb{Z}\left[\zeta_{2}\right] \rightarrow \operatorname{Pic} \mathbb{Z}\left[\zeta_{2,0}\right]$, which splits the natural map $i_{2 *}: \operatorname{Pic} \mathbb{Z}\left[\zeta_{2,0}\right] \rightarrow \mathrm{Cl} \mathbb{Z}\left[\zeta_{2}\right]$.

Proof. Clearly, Corollary 1 and Lemma 1 from $\S 1$ imply Corollary 3.

Now we are ready to obtain an explicit formula for $\operatorname{Pic} \mathbb{Z}\left[\zeta_{2,0}\right] \cong \operatorname{Pic} \mathbb{Z}\left[C_{2}\right]$. As it has been shown in $\S 1$ the following exact sequence holds:

$$
0 \rightarrow(\mathbb{Z} / p \mathbb{Z})^{(p-3) / 2+r} \rightarrow \operatorname{Pic} \mathbb{Z}\left[\zeta_{2,0}\right] \rightarrow \mathrm{Cl} \mathbb{Z}\left[\zeta_{1}\right] \oplus \mathrm{Cl} \mathbb{Z}\left[\zeta_{2}\right] \rightarrow 0
$$

where $r=\log _{p} \#\left\{\left(\operatorname{Char} S / S^{p}\right)_{\varepsilon}\right\}$.

Lemma 3. Let us denote by $L \subset S=\mathrm{Cl} \mathbb{Z}\left[\zeta_{1}\right]$ the subgroup generated by the ideal classes of $\alpha \in I\left(\mathbb{Z}\left[\zeta_{1}\right]\right)$ such that $M\left(\alpha, \mathbb{Z}\left[\zeta_{2}\right], h\right)$ is of exponent $p$ in $\operatorname{Pic} \mathbb{Z}\left[\zeta_{2,0}\right]$.

Then $L \cong\left(\operatorname{Char} S / S^{p}\right) /\left(\text { Char } S / S^{p}\right)_{\varepsilon}$.

Proof. According to our previous theory of equivalency of projective modules over $\mathbb{Z}\left[\zeta_{2,0}\right] \quad \alpha \in L \Leftrightarrow \alpha^{p}=(q) . q \equiv 1 \bmod p$. By Lemma 2 in $\S 1 q \equiv$ $1 \bmod \left(1-\zeta_{1}\right)^{p}$ and, hence, generates a character of $S / S^{p}$. Clearly, it is a determined modulo (Char $\left.S / S^{p}\right)_{\varepsilon}$.

Conversely, any element of $\left(\right.$ Char $\left.S / S^{p}\right) /\left(\text { Char } S / S^{p}\right)_{\varepsilon}$ defines $q \in Q\left(\zeta_{1}\right)$ such that $q \equiv \varepsilon \bmod \left(1-\zeta_{1}\right)^{p},(q)=\alpha^{p}$ for some $\alpha \in I\left(\mathbb{Z}\left[\zeta_{1}\right]\right)$. (See the notation on the structure of nonramified abelian extension of period $p$ over $Q\left(\zeta_{1}\right)$ in $\S 1$.) Then Corollary 1 of Lemma $2, \S 2$ implies that $M\left(\alpha^{p}, \mathbb{Z}\left[\zeta_{2}\right], h^{p}\right)$ is a free module.

Corollary 1 ("Structure of Pic $\mathbb{Z}\left[\zeta_{2,0}\right]$ "). (1) $\operatorname{Pic}^{(p)} \mathbb{Z}\left[\zeta_{2,0}\right] \cong \mathrm{Cl}^{(p)} \mathbb{Z}\left[\zeta_{2}\right] \oplus B$.

(2) $\operatorname{Ker}\left(\operatorname{Pic} \mathbb{Z}\left[\zeta_{2}, 0\right] \rightarrow \mathrm{Cl} \mathbb{Z}\left[\zeta_{1}\right] \oplus \mathrm{Cl} \mathbb{Z}\left[\zeta_{2}\right]\right) \subset B$. 
(3) $B \cong(\mathbb{Z} / p \mathbb{Z})^{(p-3) / 2} \oplus B_{1}$, where $B_{1}$ can be described as follows: $B_{1}$ is a p-group with $R$ generators, where $R=\log _{p}\left(\#\left(\operatorname{Char} S / S^{p}\right)\right)=\log _{p}\left(\#\left(S / S^{p}\right)\right)=$ (number of generators of $S$ ).

Generators of $B_{1}$ are in one-to-one correspondence with generators of $S=$ $\mathrm{Cl}^{(p)} \mathbb{Z}\left[\zeta_{1}\right]$, and if $\alpha_{1}, \ldots, \alpha_{R}$ are the generators of $S$, let us denote by $\tilde{\alpha}_{1}, \ldots$, $\tilde{\alpha}_{R}$ the correspondent generators of $B_{1}$. There are $R-r$ generators of $S$ such that $\exp \tilde{\alpha}_{i}=\exp \alpha_{i}$ and there are $r$ generators of $S$ such that $\exp \tilde{\alpha}_{i}=$ $\exp \alpha_{i}+1$, where $\exp \alpha=n$ means that $\alpha^{p^{n}}=1$ and $\alpha^{p^{n-1}} \neq 1$.

Proof. The first assertion follows from Corollary 3 of Lemma 2, §2. Obviously, $B$ is generated by all images of $M\left(\alpha, \mathbb{Z}\left[\zeta_{2}\right], h\right)$ in $\operatorname{Pic} \mathbb{Z}\left[\zeta_{2}, 0\right]$ such that $\alpha^{p^{n}}$ is a principle ideal for some $n$ and $h$ is an arbitrary element of $U\left(\mathbb{Z}\left[\zeta_{1}\right] /(p)\right)$. In the special case $n=0$ we get that $\operatorname{Ker}\left(\operatorname{Pic} \mathbb{Z}\left[\zeta_{2}, 0\right] \rightarrow \mathrm{Cl} \mathbb{Z}\left[\zeta_{1}\right] \oplus \mathrm{Cl} \mathbb{Z}\left[\zeta_{2}\right]\right)$ is contained in $B$. That proves the second statement.

The third assertion remains to be proved. By Lemma 3 there exist $R-r$ generators of $S$, namely, $\alpha_{1}, \ldots, \alpha_{R-r}$, such that $\exp \alpha_{i}=\exp M\left(\alpha_{i}, \mathbb{Z}\left[\zeta_{2}\right], h\right)$ for any $h \in U\left(\mathbb{Z}\left[\zeta_{1}\right] /(p)\right.$ ) (we identify here $\alpha_{i} \in I\left(\mathbb{Z}\left[\zeta_{1}\right]\right)$ with its image in $\mathrm{Cl} \mathbb{Z}\left[\zeta_{1}\right]$ and $M\left(\alpha_{i}, \mathbb{Z}\left[\zeta_{2}\right], h\right)$ with its image in $\left.\operatorname{Pic} \mathbb{Z}\left[\zeta_{2,0}\right]\right)$. For the remaining $r$ generators $\alpha_{R-r+1}, \ldots, \alpha_{R}$ and arbitrary $h \in U\left(\mathbb{Z}\left[\zeta_{2}\right] /(p)\right)$ we have $\exp \alpha_{i}=\exp M\left(\alpha_{i}, \mathbb{Z}\left[\zeta_{2}\right], h\right)+1$. Fix any $h_{1}, \ldots, h_{R} \in U\left(\mathbb{Z}\left[\zeta_{1}\right] /(p)\right)$, and define the subgroups $X_{1}, X_{2}, K$ in $B$ as follows:

$X_{1}$ is a subgroup generated by $M\left(\alpha_{i}, \mathbb{Z}\left[\zeta_{2}, h_{i}\right), i=1, \ldots, R-r\right.$;

$X_{2}$ is generated by $M\left(\alpha_{i}, \mathbb{Z}\left[\zeta_{2}\right], h_{i}\right), i=R-r+1, \ldots, R$;

$K=\operatorname{Ker}\left(\operatorname{Pic} \mathbb{Z}\left[\zeta_{2}, 0\right] \rightarrow \mathrm{Cl} \mathbb{Z}\left[\zeta_{1}\right] \oplus \mathrm{Cl} \mathbb{Z}\left[\zeta_{2}\right]\right)$ is generated by $M\left(\mathbb{Z}\left[\zeta_{1}\right], \mathbb{Z}\left[\zeta_{2}\right], h\right)$, where $h$ is an arbitrary element of $U\left(\mathbb{Z}\left[\zeta_{1}\right] /(p)\right)$.

Clearly, $X_{1}+X_{2}+K=B, K \cap X_{1}=0$, and $X_{1} \cap X_{2}=0$. Let $M\left(\alpha_{i}, \mathbb{Z}\left[\zeta_{2}\right], h_{i}\right)$ be a generator of $X_{2}$. Then $\left(M\left(\alpha_{i}, \mathbb{Z}\left[\zeta_{2}\right], h_{i}\right)\right)^{p^{\exp \alpha_{i}}}$ is a nontrivial element of $K$, and therefore $K \cap X_{2} \cong(\mathbb{Z} / p \mathbb{Z})^{r}$. Now we can put $B_{1}=X_{1} \oplus X_{2} \subset B$, and since $K \cong(\mathbb{Z} / p \mathbb{Z})^{(p-3) / 2+r}($ Corollary 1 , Lemma $2, \S 1)$ we obtain the required assertion.

Definition. A prime number $p$ is said to be generalized regular if any $\varepsilon \in$ $U\left(\mathbb{Z}\left[\zeta_{1}\right]\right)$, such that $\varepsilon \equiv 1 \bmod p$, satisfies the condition $p \sqrt{\varepsilon} \in \mathbb{Z}\left[\zeta_{1}\right]$.

Corollary 2. The short exact sequence

$$
0 \rightarrow(\mathbb{Z} / p \mathbb{Z})^{(p-3) / 2+r} \rightarrow \operatorname{Pic} \mathbb{Z}\left[\zeta_{2,0}\right] \rightarrow \mathrm{Cl} \mathbb{Z}\left[\zeta_{1}\right] \oplus \mathrm{Cl} \mathbb{Z}\left[\zeta_{2}\right] \rightarrow 0
$$

splits if and only if $p$ is a generalized prime number.

Proof. Obviousiy, $p$ is a generalized prime number if and only if $r=0$ and in this case $\left(\text { Char } / S / S^{p}\right)_{\varepsilon}=0$. Then Corollary 1 implies the statement.

Corollary 3 ("Structure of Pic $\mathbb{Z}\left[\zeta_{2,0}\right]$ for properly irregular prime numbers").

$$
\operatorname{Pic}^{(p)} \mathbb{Z}\left[\zeta_{2,0}\right] \cong(\mathbb{Z} / p \mathbb{Z})^{(p-3) / 2} \oplus \mathrm{Cl}^{(p)} \mathbb{Z}\left[\zeta_{2}\right] \oplus B_{1},
$$

where $B_{1}$ has $r$ generators as well as $\mathrm{Cl}^{(p)} \mathbb{Z}\left[\zeta_{1}\right]$ and they are in one-to-one correspondence with those Bernoulli numbers in the series $B_{2}, B_{4}, \ldots, B_{p-3}$, which are divisible by $p$. For each generator $\alpha_{i}$ of $\mathrm{Cl}^{(p)} \mathbb{Z}\left[\zeta_{1}\right]$ and correspondent generator $\tilde{\alpha}_{i}$ of $B_{1}$ we have $\exp \tilde{\alpha}_{i}=\exp \alpha_{i}+1$.

Proof. If $p$ is a properly irregular prime number, then $R=r$, and now our statement follows from Corollary 1. 


\section{REFERENCES}

[B] H. Bass, Algebraic $K$-theory, Benjamin, New York, 1968.

[B-Sh] Z. I. Borevich and I. R. Shafarevich, Number theory, Academic Press, New York, 1966.

[C-F] J. W. S. Cassels and A. Fröhlich, Algebraic number theory, Thompson Book Co., Washington, DC, 1967.

[C-R] C. W. Curtis and I. Reiner, Methods of representation theory, vol. II, Wiley, New York, 1987.

[G] S. Galovich, The class group of a cyclic p-group, J. Algebra 30 (1974), 368-387.

[K-M] M. A. Kervaire and M. P. Murthy, On the projective class group of cyclic groups of prime power order, Comment. Math. Helv. 52 (1977), 415-452.

[M] J. Milnor, Introduction to algebraic K-theory, Ann. of Math. Stud., vol. 72, Princeton Univ. Press, Princeton, NJ, 1971.

[S1] A. Stolin, On the $K_{0}$-group of the integer group ring of the cyclic group of order $p^{2}$, Proceeding of the All-Union Conference on the Theory of Rings, Algebras, Modules, Kishinev, 1980. (Russian)

[S2] _ On the $K_{0}$-group of the integer group ring of the cyclic group of order $p^{2}$, preprint, Kharkov University, 1980. (Russian)

[S3] _ On the Picard group of the integer group ring of a cyclic p-group and of rings close to them, preprint, Kharkov Univ., 1984. (Russian)

[U] S. Ullom, Fine structure of class groups of cyclic p-groups, J. Algebra 49 (1977), 112-124.

Department of Theoretical Mathematics, The Weizmann Institute of Science, ReHOVOT 76100 , ISRAEL

Current address: Department of Mathematics, Royal Institute of Technology, S-10044 Stockholm, Sweden

E-mail address: astolin@math.kth.se 